doi: $10.15407 /$ ujpe61.02.0168

M.YA. HORNETSKA, A.A. ROVENCHAK

Ivan Franko National University of Lviv, Chair of theoretical physics

(12, Drahomanov Str., Lviv 79005, Ukraine; e-mail: andrij.rovenchak@gmail.com)

PACS 05.30.-d, 05.30.Pr, 73.43.-f

\title{
TWO-PARAMETER MODIFICATIONS OF ANYONIC STATISTICS
}

\begin{abstract}
Two-parameter models of fractional statistics aimed at finding an expression for the occupation numbers of free anyons have been considered. Virial coefficients are found for statistics of several types: $\kappa$-deformed Polychronakos and Haldane-Wu statistics, Polychronakos and Haldane-Wu statistics modified with the q-exponential in the bosonic limit, and incomplete and nonadditive Gentile statistics for various level-filling maxima. A relation between the anyonic statistics and various statistics of fractional types is found and analyzed.

Keywords: fractional statistics, anyons, $\kappa$-deformation, Polychronakos statistics, Haldane$\mathrm{Wu}$ statistics, Gentile statistics.
\end{abstract}

\section{Introduction}

Quantum-mechanical systems described by fractional statistics have been intensively studied in the last decades. A number of approaches to this problem are known [1,2]. Methods associated with fractional statistics turned out successful, when being applied to the study of many phenomena in the physics of condensed systems, e.g., the fractional quantum Hall effect [3], high-temperature superconductivity [4], lowdimensional interacting systems $[5,6]$, as well as in such unexpected domains as dark matter models [7].

In 1924, Bose [8] derived the Planck distribution by applying the combinatory approach to light quanta. Lately, Einstein $[9,10]$ used Bose's idea and obtained a distribution for the ideal quantum gas of particles with a nonzero mass of rest.

In 1926, Dirac [11] and Fermi [12] obtained a distribution function for particles that obey the Pauli exclusion principle. In quantum-mechanical systems, the distribution is expressed as a function of energy, degeneracy order, and the number of particles in the system. For particles, whose number is unbounded in any state, the specific statistics is described by the Bose-Einstein distribution, and the corresponding particles are called bosons. If the particles obey the Pauli exclusion principle, i.e. only one particle is allowed in a certain state, the Fermi-Dirac distribution is relevant, and the particles are called fermions.

(c) M.YA. HORNETSKA, A.A. ROVENCHAK, 2016
In 1940, Gentile [13] made a first attempt to generalize those statistics. His model is characterized by a specific filling of energy levels. Namely, the number of particles on the same energy level is restricted by a finite number $s$, which is called the statistics order.

In 1977, Myrheim and Leinaas [14] showed that the phase of a wave function in a two-dimensional system can accept an arbitrary value, when two particles are swapped. Those particles were coined anyons (from the English word "any"). The term was proposed by Wilczek in 1982 [15]. The swapping of two such quasiparticles results in that $\left|\psi_{1} \psi_{2}\right\rangle=e^{i \pi \alpha}\left|\psi_{2} \psi_{1}\right\rangle$, where $\alpha$ is the parameter of anyonic statistics, which is a real number: $\alpha \in[0,1](\bmod 2)$.

Anyons compose a class of quasiparticles which are observed only in two-dimensional systems, and their properties are distinct from those of fermions and bosons. Anyons of two types are considered: Abelian and non-Abelian [16, 17]. Excitations corresponding to Abelian anyons were discovered experimentally; they play an important role in the fractional quantum Hall effect [18]. Non-Abelian anyons remain hypothetical objects, although actively studied ones.

In 1991, Haldane proposed an interpolation expression between the bosonic and fermionic limits, and introduced a generalization into the Pauli exclusion principle, which now can concern not a single, but several states [19]. In 1994, Wu [20] obtained a distribution function for the fractional exclusion statistics.

Two-parameter statistics were considered, e.g., in works $[21,22]$. It was shown there that the inter-

ISSN 2071-0194. Ukr. J. Phys. 2016. Vol. 61, No. 2 
acting gas of composite bosons (consisting of two fermions or two bosons) can be algebraically realized with the help of the model of deformed Bose gas with a structure function that is a combination of a $q$-deformation and a quadratic-polynomial deformation. In this work, the applied approach is based on work [23], where the two-parameter fractional statistics were used to model anyons.

The expression for the occupation numbers in an ideal gas of anyons still remains unknown. This circumstance complicates the analysis of the thermodynamics of anyons, and a statistical mechanical model which would correspond to anyons is also absent [1]. The application of the two-parameter statistics allows systems to be effectively described with an accuracy higher than conventional one-parameter statistics do. Moreover, the considered models can be applied to the studies of interacting quantum gases $[5,24]$.

In Section 2, the general relations for virial and cluster expansions, as well as the virial coefficients for anyons, are considered in brief. In Section 3, statistics modifications are analyzed, and the results of numerical calculations of virial coefficients for six statistics are reported. In addition, a modified Polychronakos statistics with a $q$-exponential is analyzed in the bosonic limit. Section 4 is devoted to finding the relations between the parameters of anyonic statistics and fractional statistics of other types. The results obtained are summarized in Conclusions.

\section{Virial and Cluster Expansions}

\subsection{General relations}

The virial expansion for the equation of state of a two-dimensional particle system can be written in the form

$\frac{p}{T}=\rho_{2}\left[1+b_{2}\left(\rho_{2} \lambda^{2}\right)+b_{3}\left(\rho_{2} \lambda^{2}\right)^{2}+\ldots\right]$,

where $p$ is the pressure, $T$ the absolute temperature, $\rho_{2}=\frac{N}{A}$ is the two-dimensional density (concentration) of the system, and

$\lambda=\left(\frac{2 \pi \hbar^{2}}{m T}\right)^{1 / 2}$

is the thermal de Broglie wavelength. The factors $b_{j}$ $(j=2,3, \ldots)$ are the dimensionless $j$-th virial coefficients. Let us recall the form of the virial expansion

ISSN 2071-0194. Ukr. J. Phys. 2016. Vol. 61, No. 2 for the ideal two-dimensional gas obeying the Fermi or Bose statistics [1]:

$\frac{p}{T}=\rho_{2}\left(1 \pm \frac{1}{4} \rho_{2} \lambda^{2}+\ldots\right)$

Here, the upper sign corresponds to fermions (F), and the lower one to bosons (B). It is easy to show that the second virial coefficients are as follows:

$b_{2}^{\mathrm{F}}=+\frac{1}{4}, \quad b_{2}^{\mathrm{B}}=-\frac{1}{4}$.

Let us use the cluster expansion to determine the virial coefficients. The equation of state in terms of the grand partition function $\Xi$ looks like

$\frac{p}{T}=\frac{1}{A} \ln \Xi(z, V, T)$,

where $z$ is the fugacity, which is related to the chemical potential $\mu$ by the formula $z=e^{\mu / T}$. The density equals

$\rho_{2}=\frac{N}{A}=z \frac{\partial}{\partial z}\left(\frac{1}{A} \ln \Xi\right)_{A, T}=T \frac{\partial}{\partial \mu}\left(\frac{1}{A} \ln \Xi\right)_{A, T}$.

Applying the cluster expansion

$\frac{p}{T}=\frac{1}{A} \ln \Xi=\sum_{\ell=1}^{\infty} B_{\ell} z^{\ell}$

to the equation of state, we obtain

$\sum_{\ell=1}^{\infty} B_{\ell} z^{\ell}=\left(\sum_{\ell=1}^{\infty} \ell B_{\ell} z^{\ell}\right)\left[1+b_{2} \lambda^{2}\left(\sum_{\ell=1}^{\infty} \ell B_{\ell} z^{\ell}\right)+\ldots\right]$.

From whence, we can find a relation between the virial and cluster coefficients.

The second, third, and fourth virial coefficients look like [25]

$$
\begin{aligned}
& b_{2} \lambda^{2}=-\frac{B_{2}}{B_{1}^{2}}, \quad b_{3} \lambda^{4}=-2 \frac{B_{3}}{B_{1}^{3}}+4 \frac{B_{2}^{2}}{B_{1}^{4}}, \\
& b_{4} \lambda^{6}=-3 \frac{B_{4}}{B_{1}^{4}}+18 \frac{B_{2} B_{3}}{B_{1}^{4}}-20 \frac{B_{2}^{3}}{B_{1}^{6}} .
\end{aligned}
$$

Expressions for higher virial coefficients can be analogously obtained by equating the coefficients in the terms with identical power exponents of the variable $z$. 


\subsection{Virial coefficients of anyons}

While finding the virial coefficients of anyons, we can use the fact that the grand partition function can be written as an infinite series of statistical sums $Z_{N}$, each for a system of $N$ particles:

$\Xi=\sum_{N=0}^{\infty} z^{N} Z_{N}, \quad Z_{0} \equiv 1$.

From the cluster expansion (7), we obtain the cluster integrals in terms of partition functions [1]:

$$
\begin{aligned}
B_{1} & =\frac{Z_{1}}{A}, \quad B_{2}=\frac{2 Z_{2}-Z_{1}^{2}}{2 A}, \\
B_{3} & =\frac{3 Z_{3}-3 Z_{2} Z_{1}+Z_{1}^{3}}{3 A}, \ldots
\end{aligned}
$$

With the help of formulas (8) and (10), the second virial coefficient of the ideal anyon gas can be calculated:

$b_{2}=-\frac{A}{\lambda^{2}} \frac{\left(2 Z_{2}-Z_{1}^{2}\right)}{2 Z_{1}^{2}}$.

Below, the ideal anyon gas is regarded as an interacting Bose gas [1]. From this viewpoint, let us rewrite expression (11) in the form of two terms,

$b_{2}(\alpha)=b_{2}(0)-\frac{A}{\lambda^{2}} \frac{Z_{2}(\alpha)-Z_{2}(0)}{Z_{1}^{2}}$,

where the parameters $b_{2}(0)$ and $Z_{2}(0)$ correspond to the ideal Bose gas, and the parameter $\alpha \in[0,1]$. Here, we used the fact that the one-particle partition function $Z_{1}$ does not depend on the statistics, i.e. it is identical for fermions, bosons, and anyons. It is worth noting that all expressions are considered only in the thermodynamic limit $A \rightarrow \infty$.

For convenience, let the system be in an oscillator potential with the frequency $\omega$ playing the regulator role. The spectrum obtained in the one-particle problem, $E_{n}=(n+1) \hbar \omega$, and the spectrum obtained in the one-anyon problem, which has two branches [1]:

- an $(n+1)$-fold degenerate one with $E_{n}^{(1)}=(2 n+$ $+1+\alpha) \hbar \omega$ and

- an $n$-fold degenerate one with $E_{n}^{(1)}=(2 n+1-$ $-\alpha) \hbar \omega$,

where $n=0,1,2,3, \ldots$, should be taken into account. Then, after some transformations, we obtain an exact expression for the second virial coefficient of the ideal anyon gas $[1,26]$

$b_{2}^{\text {anyon }}(\alpha)=-\frac{1}{4}\left(1-4 \alpha+2 \alpha^{2}\right)$,

where the second virial coefficient of the ideal Bose gas $b_{2}(0)=-1 / 4$ [see Eq. (4)] was used. An interesting fact is that, at $\alpha=1$, we obtain a correct fermionic limit (4).

Unfortunately, the problem with a larger number of anyons cannot be solved exactly. However, the results of numerical calculations are available for the higher virial coefficients. In particular, the third coefficient for anyons looks like [26]

$b_{3}^{\text {anyon }}(\alpha)=\frac{1}{36}+\frac{\sin ^{2} \pi \alpha}{12 \pi^{2}}+c_{3} \sin ^{4} \pi \alpha$,

where

$c_{3}=-(1.652 \pm 0.012) \times 10^{-5}$.

The fourth virial coefficient for anyons equals

$b_{4}^{\text {anyon }}(\alpha)=\frac{\sin ^{2} \pi \alpha}{16 \pi^{2}}\left(\frac{\ln (\sqrt{3}+2)}{\sqrt{3}}+\cos \pi \alpha\right)+$

$+\left(c_{4}+d_{4} \cos \pi \alpha\right) \sin ^{4} \pi \alpha$,

where

$c_{4}=-0.0053 \pm 0.0003, \quad d_{4}=-0.0048 \pm 0.0009$.

\section{Statistics Modifications}

\subsection{Expressions for occupation numbers}

Let us consider modifications of fractional statistics on the basis of three following general expressions for occupation numbers [23]. The first expression,

$n_{j}^{\mathrm{P}}=\frac{1}{z^{-1} X\left(\varepsilon_{j}\right)+Y}$,

generalizes the standard Bose (Fermi) statistics at $X\left(\varepsilon_{j}\right)=e^{\frac{\varepsilon_{j}}{T}}$ and $Y=-1(Y=+1)$, and defines the Polychronakos fractional statistics [28] at $Y=-\gamma=$ const $\neq \pm 1$. The second expression is the modified Haldane-Wu statistics $[19,20]$, which is presented in the form

$n_{j}^{\mathrm{HW}}=\frac{1}{w\left[z^{-1} X\left(\varepsilon_{j}\right)\right]+g}$.

ISSN 2071-0194. Ukr. J. Phys. 2016. Vol. 61, No. 2 
Here, the function $w(\xi)$ is a solution of the transcendental equation

$w^{g}(1+w)^{1-g}=\xi$,

which can be obtained with the help of a certain expression for the number of microstates in a manybody quantum-mechanical system providing an interpolation between bosons and fermions. It is easy to be convinced that we obtain $w(\xi)=\xi-1$, i.e. the Bose distribution, at $g=0$ and $w(\xi)=\xi$, i.e. the Fermi distribution, at $g=1$. In the limit $\xi \rightarrow \infty$, the latter equation has the solution $w(\xi) \simeq \xi$. It gives rise to the Boltzmann distribution

$n_{j}=e^{-\left(\varepsilon_{j}-\mu\right) / T}$,

which does not depend on the statistics parameter $g$. The third expression is the Gentile statistics [13]

$n_{j}^{\mathrm{G}}=\frac{1}{z^{-1} X\left(\varepsilon_{j}\right)-1}-\frac{(s+1)}{z^{-(s+1)} X^{(s+1)}\left(\varepsilon_{j}\right)-1}$.

One can easily be convinced that we have the Fermi distribution at $s=1$ and the Bose one at $s=\infty$. In addition to the parameters $\gamma, \kappa$, or $s$, another deformation parameter can be introduced into each of those models [23].

In this work, we consider only the following modifications: the $\kappa$-exponential appears instead of the ordinary Boltzmann factor $e^{\varepsilon / T}$ in $\kappa$-deformed statistics, and the factor $e^{q x}$ instead of $e^{x}$ in incomplete statistics $[29,30]$. We will also consider two variants of the so-called $q$-exponentials.

The $\kappa$-exponential is written in the form $[31,32]$

$\exp _{\kappa}(x)=\left(\sqrt{1+\kappa^{2} x^{2}}+\kappa x\right)^{1 / \kappa}$.

The series expansions of Eqs. (16), (17), and (20) in the activity $z$ read [33]

$n_{j}^{\mathrm{P}}=\sum_{l=1}^{\infty}(-1)^{l-1} \frac{Y^{l-1}}{X^{l}} z^{l}=$

$=\frac{1}{X} z-\frac{Y}{X^{2}} z^{2}+\frac{Y^{2}}{X^{3}} z^{3} \mp \ldots$,

$n_{j}^{\mathrm{HW}}=\sum_{m=0}^{\infty}(-1)^{m} \frac{\Gamma[g(m+1)]}{n ! \Gamma[g(m+1)-m]} \frac{z^{m+1}}{X^{m+1}}=$

$=\frac{1}{X} z-\frac{(2 g-1)}{X^{2}} z^{2}+\frac{(3 g-2)(3 g-1)}{2 ! X^{3}} z^{3} \mp \ldots$,

$$
\begin{aligned}
& n_{i}^{\mathrm{G}}=\sum_{l=1}^{\infty}(-1)^{l-1} \frac{z^{l}}{X^{l}}-(s+1) \sum_{l=1}^{\infty}(-1)^{l-1} \frac{z^{l(s+1)}}{X^{l(s+1)}}= \\
& =\left(\frac{1}{X} z-\frac{1}{X^{2}} z^{2}+\frac{1}{X^{3}} z^{3} \mp \ldots\right)- \\
& -(s+1)\left(\frac{1}{X^{s+1}} z^{(s+1)}-\frac{1}{X^{2(s+1)}} z^{2(s+1)} \pm \ldots\right) .
\end{aligned}
$$

For simplicity, the summation over the levels, $\sum_{j}$, in the equations

$$
N=\sum_{j} g_{j} n_{j}, \quad \frac{N}{A}=\frac{1}{A} \sum_{j} g_{j} n_{j}=\sum_{l=1}^{\infty} l B_{l} z^{l}
$$

is substituted by the integration over the energy. The density of states of the function $G(\varepsilon)$ in a twodimensional ideal gas of particles with the mass $m$ equals $G(\varepsilon)=\frac{m A}{2 \pi \hbar^{2}}=$ const [34] (cf. also work [1, p. 150] or [35, p. 22]), so that

$$
\sum_{j} \ldots=\int_{0}^{\infty} d \varepsilon G(\varepsilon) \ldots
$$

Using various representations for the function $X$, the cluster integrals $B_{l}$ can be easily calculated, by using expansions (22)-(24), and virial coefficients are obtained from Eqs. (8). Below, we present the results of calculations for virial coefficients in some statistics.

\subsection{Results for Virial Coefficients}

- $\kappa$-deformed Polychronakos statistics $(\kappa \mathrm{PS})$. For the statistics of this type,

$X(\varepsilon)=e_{\kappa}^{\frac{\varepsilon}{T}}=\left(\sqrt{1+\kappa^{2} \frac{\varepsilon^{2}}{T^{2}}}+\kappa \frac{\varepsilon}{T}\right)^{1 / \kappa}, \quad Y=-\gamma$.

The cluster integrals equal

$$
\begin{aligned}
& B_{1} \lambda^{2}=-\frac{1}{\kappa^{2}-1}, \quad B_{2} \lambda^{2}=-\frac{\gamma}{\kappa^{2}-4}, \\
& B_{3} \lambda^{2}=-\frac{\gamma^{2}}{\kappa^{2}-9}, \ldots
\end{aligned}
$$

The second and third virial coefficients are

$$
\begin{aligned}
& b_{2}^{\kappa \mathrm{PS}}=-\gamma \frac{\left(\kappa^{2}-1\right)^{2}}{\left(\kappa^{2}-4\right)} \\
& b_{3}^{\kappa \mathrm{PS}}=2 \gamma^{2}\left(\kappa^{2}-1\right)^{4}\left[\frac{2}{\left(\kappa^{2}-4\right)^{2}}-\frac{1}{\left(\kappa^{2}-9\right)\left(\kappa^{2}-1\right)}\right] .
\end{aligned}
$$


- $\kappa$-deformed Haldane-Wu statistics $(\kappa \mathrm{HWS})$. For the statistics of this type,

$X(\varepsilon)=e_{\kappa}^{\frac{\varepsilon}{T}}=\left(\sqrt{1+\kappa^{2} \frac{\varepsilon^{2}}{T^{2}}}+\kappa \frac{\varepsilon}{T}\right)^{1 / \kappa}$.

The cluster integrals equal

$$
\begin{aligned}
& B_{1} \lambda^{2}=-\frac{1}{\kappa^{2}-1}, \quad B_{2} \lambda^{2}=\frac{(2 g-1)}{\kappa^{2}-4}, \\
& B_{3} \lambda^{2}=-\frac{(3 g-2)(3 g-1)}{2 !\left(\kappa^{2}-9\right)}, \ldots
\end{aligned}
$$

The second and third virial coefficients are $b_{2}^{\kappa \mathrm{HWS}}=-(2 g-1) \frac{\left(\kappa^{2}-1\right)^{2}}{\kappa^{2}-4} ;$ $b_{3}^{\kappa \mathrm{HWS}}=\left(\kappa^{2}-1\right)^{4}\left[\frac{4(2 g-1)^{2}}{\left(\kappa^{2}-4\right)^{2}}-\frac{(3 g-2)(3 g-1)}{\left(\kappa^{2}-9\right)\left(\kappa^{2}-1\right)}\right]$.

- Incomplete Gentile statistics (IGS). For the statistics of this type,

$X=e^{\frac{q \varepsilon}{T}}$.

Let us consider the cluster integrals and the second and third virial coefficients for various $s$-values.

$\star \mathrm{s}=\mathbf{2}$ :

The cluster integrals equal

$B_{1} \lambda^{2}=\frac{1}{q}, \quad B_{2} \lambda^{2}=\frac{1}{4 q}, \quad B_{3} \lambda^{2}=-\frac{2}{9 q}, \ldots$

The second and third virial coefficients are

$b_{2}^{\mathrm{IGS} 2}=-\frac{q}{4} ; \quad b_{3}^{\mathrm{IGS} 2}=\frac{25}{36} q^{2}$.

$\star \mathbf{s}=\mathbf{3}$ :

The cluster integrals equal

$B_{1} \lambda^{2}=\frac{1}{q}, \quad B_{2} \lambda^{2}=\frac{1}{4 q}, \quad B_{3} \lambda^{2}=\frac{1}{9 q}, \ldots$

The second and third virial coefficients are

$$
b_{2}^{\mathrm{IGS} 3}=-\frac{q}{4} ; \quad b_{3}^{\mathrm{IGS} 3}=\frac{q^{2}}{36} .
$$

$\star s=4:$

The cluster integrals equal

$B_{1} \lambda^{2}=\frac{1}{q}, \quad B_{2} \lambda^{2}=\frac{1}{4 q}, \quad B_{3} \lambda^{2}=\frac{1}{9 q}, \ldots$

172
The second and third virial coefficients are

$b_{2}^{\mathrm{IGS} 4}=-\frac{q}{4} ; \quad b_{3}^{\mathrm{IGS} 4}=\frac{q^{2}}{36}$.

- Nonadditive Gentile statistics (NGS). For the statistics of this type, we used the so-called Tsallis $q$-exponential $e_{q}^{x}[36]$. It equals

$e_{q}^{x}=[1+(1-q) x]^{\frac{1}{1-q}}$,

if the quantity in the square brackets is larger than zero and zero $\left(e_{q}^{x}=0\right)$ otherwise. Then,

$X(\varepsilon)=e_{q}^{\frac{\varepsilon}{T}}=\left[1+(1-q) \frac{\varepsilon}{T}\right]^{\frac{1}{1-q}}$.

Let us consider the cluster integrals and the second and third virial coefficients for various $s$-values.

$\star \mathrm{s}=\mathbf{2}$ :

The cluster integrals equal

$B_{1} \lambda^{2}=\frac{1}{q}, \quad B_{2} \lambda^{2}=\frac{1}{2(1+q)}$,

$B_{3} \lambda^{2}=-\frac{2}{3 q(2+q)}, \ldots$.

The second and third virial coefficients are

$b_{2}^{\mathrm{NGS} 2}=-\frac{q^{2}}{2(1+q)} ;$

$b_{3}^{\mathrm{NGS} 2}=\frac{q^{2}}{4}\left[\frac{16}{3(2+q)}+\frac{1}{(1+q)^{2}}\right]$.

$\star \mathrm{s}=3$ :

The cluster integrals equal

$B_{1} \lambda^{2}=\frac{1}{q}, \quad B_{2} \lambda^{2}=\frac{1}{2(1+q)}$,

$B_{3} \lambda^{2}=\frac{1}{3(2+q)}, \ldots$.

The second and third virial coefficients are

$b_{2}^{\mathrm{NGS} 3}=-\frac{q^{2}}{2(1+q)} ;$

$b_{3}^{\mathrm{NGS} 3}=q^{4}\left[\frac{1}{(1+q)^{2}}-\frac{2}{3 q(2+q)}\right]$.

$\star s=4:$

The cluster integrals equal

$B_{1} \lambda^{2}=\frac{1}{q}, \quad B_{2} \lambda^{2}=\frac{1}{2(1+q)}$,

$B_{3} \lambda^{2}=\frac{1}{3(2+q)}, \ldots$

ISSN 2071-0194. Ukr. J. Phys. 2016. Vol. 61, No. 2 
The second and third virial coefficients are

$$
\begin{aligned}
& b_{2}^{\mathrm{NGS} 4}=-\frac{q^{2}}{2(1+q)} ; \\
& b_{3}^{\mathrm{NGS} 4}=q^{4}\left[\frac{1}{(1+q)^{2}}-\frac{2}{3 q(2+q)}\right] .
\end{aligned}
$$

It is clear that the Gentile statistics with $s \geq 4$ coincides with the Bose statistics at least to an accuracy of the fourth virial coefficient.

\section{3. q-exponential in the bosonic limit of Polychronakos and Haldane-Wu statistics}

In the problems dealing with the so-called $q$-deformed commutators

$$
[A, B]_{q}=A B-q B A,
$$

there arise deformed exponentials of a different type as compared with those considered in the previous sections [37]. We use the notation

$E_{q}^{x}=\sum_{j=0}^{\infty} \frac{x_{j}}{[j]_{q} !}$

for them, where the $q$-factorial is defined by the formula

$[j]_{q} !=[j]_{q}[j-1]_{q} \ldots[1]_{q}$,

and the notation $[n]_{q}$ stands for the $q$-analog of the number $n$,

$[n]_{q}=\frac{q^{n}-1}{q-1}=1+q+q^{2}+\ldots+q^{n-1}$.

The general formula for the series expansion of the $q$-exponential looks like

$$
\begin{aligned}
& E_{q}^{x}=\sum_{n=0}^{\infty} x^{n} \frac{(q-1)^{n}}{\left(q^{n}-1\right)\left(q^{n-1}-1\right) \ldots(q-1)}= \\
& =\sum_{n=0}^{\infty} x^{n} \frac{q-1}{q^{n}-1} \frac{q-1}{q^{n-1}-1} \ldots \frac{q-1}{q^{2}-1} \frac{q-1}{q-1} .
\end{aligned}
$$

Therefore, $E_{q}^{x}$ can be written in the form

$$
E_{q}^{x}=\sum_{n=0}^{\infty} x^{n} \frac{1}{\left(1+\ldots+q^{n-1}\right)\left(1+\ldots+q^{n-2}\right) \ldots(1+q) 1} .
$$

ISSN 2071-0194. Ukr. J. Phys. 2016. Vol. 61, No. 2
In the limit $q \rightarrow 1$, we expand the function $E_{q}^{x}$ in a series

$E_{q}^{x}=f_{0}(x)+(q-1) f_{1}(x)+(q-1)^{2} f_{2}(x)+\ldots$.

Substituting $q=1$, we obtain the following expression for $f_{0}(x)$ :

$f_{0}(x)=\sum_{n=0}^{\infty} x^{n} \frac{1}{n !}=e^{x}$.

It can also be demonstrated that the next expansion terms give the following coefficient functions:

$$
\begin{aligned}
& f_{1}(x)=-\frac{x^{2}}{4} e^{x}, \\
& f_{2}(x)=\frac{x^{2}}{8}\left[1+\frac{17}{9} x+\frac{59}{36} x^{2}+\frac{31}{36} x^{3}+\frac{17}{54} x^{4}+\right. \\
& \left.+\frac{47}{540} x^{5}+\frac{83}{4320} x^{6}+\ldots\right] .
\end{aligned}
$$

The Polychronakos statistics modified with this exponential is defined as follows:

$n_{j}^{q \mathrm{P}}=\frac{1}{z^{-1} E_{q}^{\varepsilon_{j} / T}-\gamma}$.

The corresponding Haldane-Wu statistics looks like

$n_{j}^{q \mathrm{HW}}=\frac{1}{w\left[z^{-1} E_{q}^{\varepsilon_{j} / T}\right]+g}$.

In the next section, those statistics will be analyzed in more details.

\section{Relationship between the Anyonic and Other Statistics}

Having described the statistics and having obtained expressions for virial coefficients, we can establish the correspondence between the anyonic statistics and the fractional statistics of other types. For this purpose, the following procedure has to be done. We equate the second and third virial coefficients for the anyonic and other corresponding statistics to obtain a system of equations

$\left\{\begin{array}{l}b_{2}=b_{2}^{\text {anyon }}, \\ b_{3}=b_{3}^{\text {anyon }}\end{array}\right.$,

Hence, we have two equations for two parameters $(\kappa, \gamma, \ldots)$. Having calculated those statistical parameters $(\kappa, \gamma, \ldots)$ at various anyonic parameters $\alpha \in[0,1]$, 

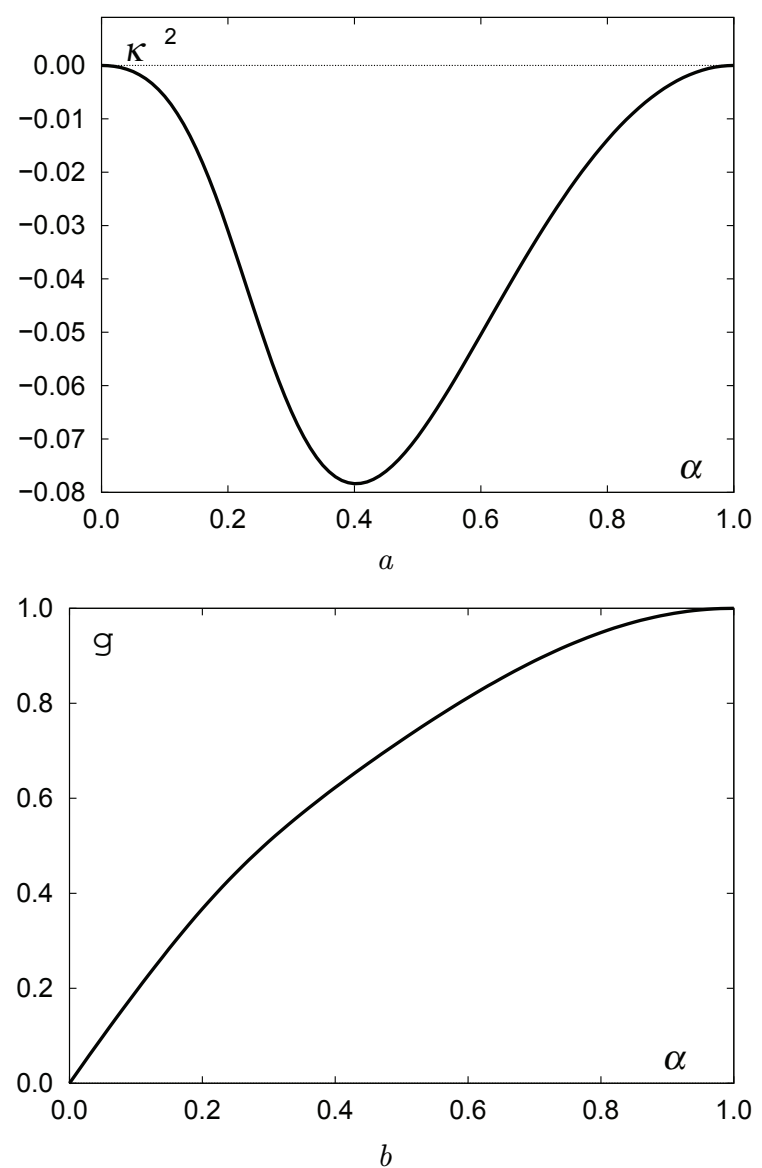

Fig. 1. Parameters $\kappa^{2}(a)$ and $g(b)$ as functions of the anyonic parameter $\alpha$ in the $\kappa$-deformed Haldane-Wu statistics

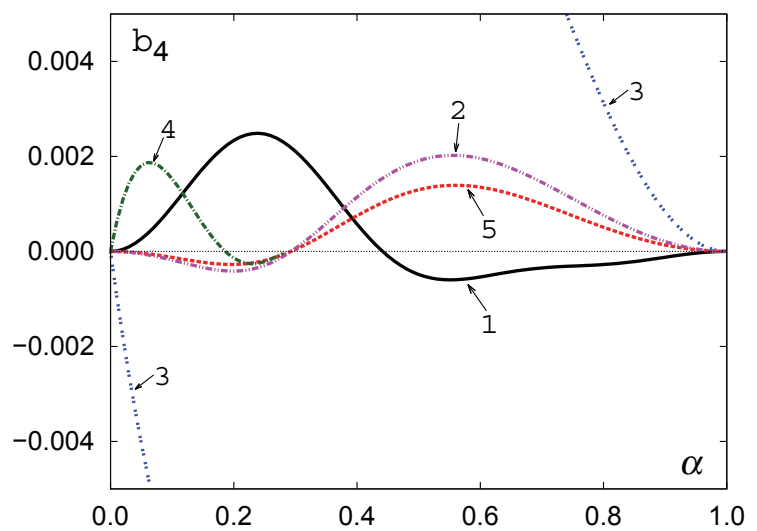

Fig. 2. Fourth virial coefficient in various statistics: anyons (1), $\kappa$-deformed Haldane-Wu statistics (2), $\kappa$-deformed Polychronakos statistics (3), Gentile statistics with $s>3$ (equivalent to the Bose statistics) (4), Haldane-Wu statistics deformed with the Tsallis exponential [23] (5) we can find a value for the fourth virial coefficient, which will demonstrate how much the proposed twoparameter statistics differ from the anyonic one.

Numerical calculations testify that the substitution of the Tsallis $q$-exponential by the $\kappa$-exponential brings about the results that qualitatively coincide with those obtained in work [23] dealing with a modification of the Polychronakos and Haldane-Wu statistics. It turns out that, in this case, $\kappa^{2}<0$, i.e. the parameter $\kappa$ has an imaginary value. However, this result is not of surprise, because complex parameters can arise in fractional statistics in various contexts [34, 38, 39].

Figure 1 demonstrates the dependences of the parameters $\kappa^{2}$ and $g$ on the anyonic parameter $\alpha$ in the $\kappa$-deformed Haldane- $\mathrm{Wu}$ statistics.

In Fig. 2, the fourth virial coefficient is compared in various fractional statistics. It is of interest that, in all analyzed statistics, the behavior of $b_{4}$ differs qualitatively from the result obtained for anyons. Namely, they are of different signs in the bosonic limit. The only exception is the Gentile statistics with $s=4$ and the Tsallis $q$-exponential, which is, in fact, the deformed Bose statistics.

In the case of intermediate distribution, which corresponds to the Gentile statistics, where the maximum occupation number of the levels is confined by the number $s$, we considered the values $s=2,3$, and 4. Actually, each of the corresponding expressions for the occupation number is a separate distribution, in which the exponential deformation gives one parameter. We associated it with the anyonic $\alpha$ by equating the second virial coefficients,

$b_{2}=b_{2}^{\text {anyon }}$.

In this case, the third virial coefficient differs from the anyonic one, as is shown in Fig. 3. However, as was said above, the behavior of the fourth virial coefficient at $s>3$ qualitatively coincides with the anyonic one, which gives grounds to consider this model as a basis for further modifications.

Now, let us consider numerical results for the Polychronakos statistics with the $q$-exponential $E_{q}^{x}$ (48). Expanding expression (56) in a $z$-series, we obtain the following formulas for cluster integrals with a second-order accuracy in small corrections $\theta$ and $\eta$ $(q=1+\theta$ and $\gamma=1+\eta)$ :

$B_{1}=1+\frac{1}{2} \eta+\eta^{2} P_{1}$,

ISSN 2071-0194. Ukr. J. Phys. 2016. Vol. 61, No. 2 


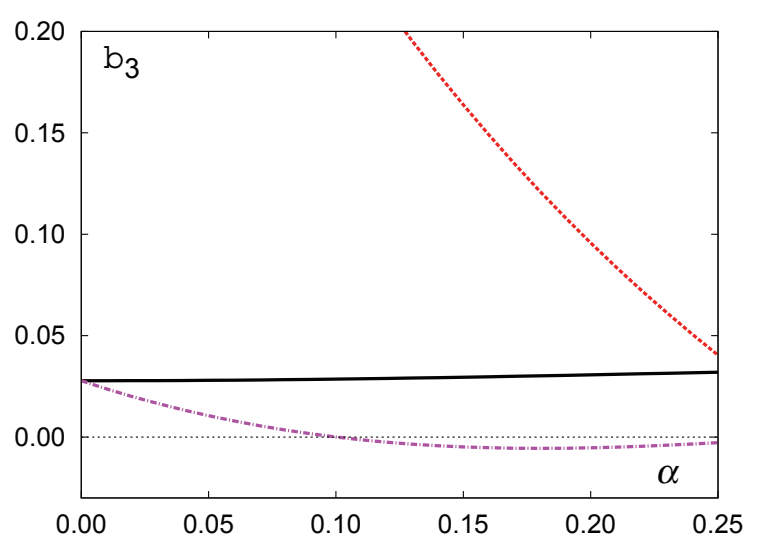

Fig. 3. Third virial coefficient in various variants of the Gentile statistics with the Tsallis exponential: $s=2$ (dashed curve), $s>2$ (dash-dotted curve), anyons (solid curve)

$$
\begin{aligned}
& B_{2}=\frac{1}{2}\left[\frac{1}{2}+\frac{1}{2} \theta+\frac{1}{8} \eta+\frac{1}{8} \eta \theta+\eta^{2}\left(\frac{9}{64}+P_{2}\right)\right], \\
& B_{3}=\frac{1}{3}\left[\frac{1}{3}+\frac{2}{3} \theta+\frac{1}{3} \theta^{2}+\frac{1}{18} \eta+\frac{1}{9} \eta \theta+\eta^{2}\left(\frac{1}{27}+P_{3}\right)\right], \\
& B_{4}=\frac{1}{4}\left[\frac{1}{4}+\frac{3}{4} \theta+\frac{3}{4} \theta^{2}+\frac{3}{32} \eta \theta+\eta^{2}\left(\frac{15}{1024}+P_{4}\right)\right],
\end{aligned}
$$

where

$$
\begin{aligned}
& P_{j}=-j \int_{0}^{\infty} e^{-(j+1) x} f_{2}(x) d x, \\
& P_{1}=-1.09993 \ldots, \quad P_{2}=-0.181866 \ldots, \\
& P_{3}=-0.272799 \ldots, \quad P_{4}=-0.0813217 \ldots .
\end{aligned}
$$

Whence the virial coefficients are

$$
\begin{aligned}
& b_{2}^{q \mathrm{P}}=-\frac{1}{4}-\frac{\theta}{4}+\frac{3 \eta}{16}+\left(\frac{3}{16} \theta \eta-0.654347 \eta^{2}\right) \\
& b_{3}^{q \mathrm{P}}=\frac{1}{36}+\frac{\theta}{18}-\frac{17}{216} \eta+\left(\frac{\theta^{2}}{36}-\frac{17}{108} \theta \eta+0.595426 \eta^{2}\right) .
\end{aligned}
$$

In Fig. 4, the dependences of the corrections $\theta$ and $\eta$ on the anyonic parameter $a$ are shown. It should be noted that those corrections are small only in a close vicinity of the bosonic limit $\alpha \rightarrow 0$.

In Fig. 5, the dependence of the fourth virial coefficient in the Polychronakos statistics modified with $q$-exponential on the anyonic parameter $\alpha$ is exhibited.

ISSN 2071-0194. Ukr. J. Phys. 2016. Vol. 61, No. 2

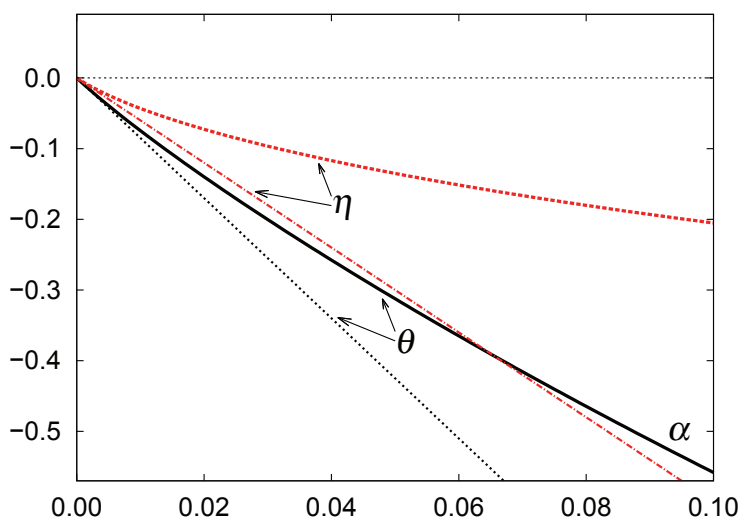

Fig. 4. Dependences of small corrections on the anyonic parameter in the Polychronakos statistics with $q$-exponential (48)

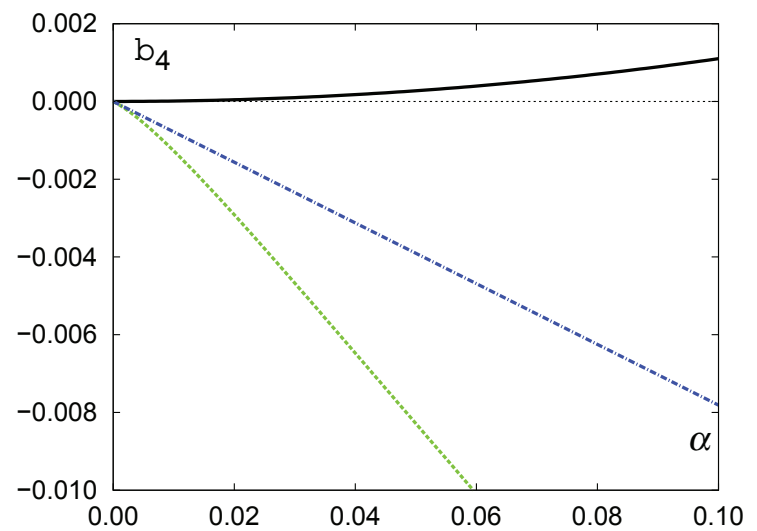

Fig. 5. Fourth virial coefficient in the Polychronakos statistics with $q$-exponential (48): result for anyons (solid curve), linear approximation in $\theta$ and $\eta$ (dash-dotted curve), quadratic approximation in $\theta$ and $\eta$ (dashed curve)

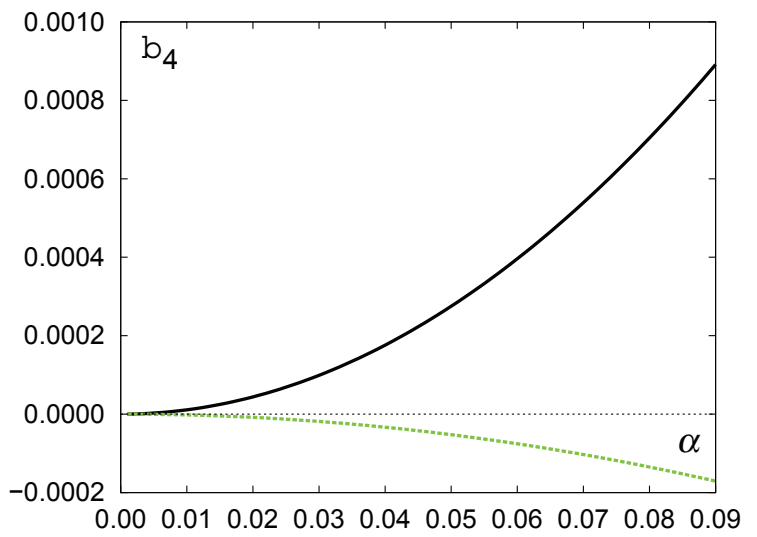

Fig. 6. Fourth virial coefficient in the Haldane-Wu statistics with $q$-exponential (48): result for anyons (solid curve), quadratic approximation in $g$ and $\theta$ (dashed curve) 
Carrying out similar calculations for the HaldaneWu statistics with $q$-exponential $E_{q}^{x}$ (48) and expanding in the small parameter $g$ and the small deviation $\theta=q-1$, we obtain

$b_{2}^{q \mathrm{HW}}=-\frac{1}{4}+\frac{g}{2}+0.00144676 \theta-$

$-0.00289352 g \theta-0.459052 \theta^{2}$,

$b_{3}^{q \mathrm{HW}}=\frac{1}{36}+0.00145216 \theta-$

$-0.00798148 g \theta+0.225433 \theta^{2}$.

A comparison of the fourth virial coefficient in this statistics with the anyonic one is shown in Fig. 6 .

Figures 5 and 6 testify that the fourth virial coefficient in two last considered statistics has a sign that is opposite to the sign of anyonic $b_{4}^{\text {anyon }}$. Despite that, the results obtained in this work, similarly to those of work [23], provide the reproduction of thermodynamic functions for anyons with an accuracy that exceeds the available accuracy of experimental measurements [40].

\section{Conclusions}

In this work, fractional statistics generalizing the quantum-mechanical Bose-Einstein and Fermi-Dirac distributions are considered. Information is reported concerning the virial and cluster expansions and the virial coefficients for anyons. Attention is focused on the modifications of three statistics, namely, the Polychronakos, Haldane- $\mathrm{Wu}$, and Gentile ones.

Cluster integrals and virial coefficients are determined for statistics of seven types: incomplete and $\kappa$-deformed Polychronakos statistics, incomplete and $\kappa$-deformed Haldane-Wu statistics, and incomplete and non-additive Gentile statistics for various $s$-values. The modified Polychronakos statistics with the $q$-exponential was also considered in the bosonic limit.

Numerical results are reported for the following statistics: $\kappa$-deformed Haldane-Wu and Polychronakos statistics; Gentile statistics with the Tsallis exponential at $s=2,3$, and 4 ; and small corrections to the Polychronakos statistics with the $q$-exponential. It is shown that none of the generalized statistics gives an exact correspondence with the anyonic one [1]. Only some of them can be partially compared with the latter, and only with a confined accuracy to the third virial coefficient, inclusive, which confirms the previous results [23].

The obtained conclusions open some prospects for further studies of the problem concerned in several directions. In particular, these are the study of two-parameter modifications of the Bose (Gentile at $s>3$ ) statistics and the construction of an expression for the occupation numbers as a series in small corrections.

The work was partially sponsored by the State Fund for Fundamental Researches of Ukraine (project F64/41-2015, state registration No. 0115U004838).

1. A. Khare, Fractional Statistics and Quantum Theory (World Scientific, Singapore, 2005).

2. A. Rovenchak, Zh. Fiz. Dosl. 172, 2001 (2013).

3. G.S. Jeon and J.K. Jain, Phys. Rev. B 81, 035319 (2010).

4. G.S. Canright and M.D. Johnson, J. Phys. A 27, 3579 (1994).

5. M.T. Batchelor, X.W. Guan, and N. Oelkers, Phys. Rev. Lett. 96, 210402 (2006).

6. D.-V. Anghel, Phys. Scr. T 151, 014079 (2012).

7. Z. Ebadi, B. Mirza, and H. Mohammadzadeh, J. Cosmol. Astropart. Phys. 2013, 057 (2013).

8. S.N. Bose, Z. Phys. 26, 178 (1924).

9. A. Einstein, Sitzungsber. Preuss. Konigl. Akad. Wiss. phys.-math. Klasse, 261 (1924).

10. A. Einstein, Sitzungsber. Preuss. Konigl. Akad. Wiss. phys.-math. Klasse, 3 (1925).

11. P.A.M. Dirac, Proc. R. Soc. London A 112, 661 (1926).

12. E. Fermi, Z. Phys. 36, 902 (1926).

13. G. Gentile, Nuovo Cimento 17, 493 (1940).

14. J.M. Leinaas and J. Myrheim, Nuovo Cimento 37B, 1 (1977).

15. F. Wilczek, Phys. Rev. Lett. 49, 957 (1987).

16. A.P. Polychronakos, Phys. Rev. Lett. 84, 1268 (2000).

17. F. Mancarella, A. Trombettoni, and G. Mussardo, Nucl. Phys. B 867 [FS], 950 (2013).

18. F.E. Camino, W. Zhou, and V.J. Goldman, Phys. Rev. B 72, 155313 (2005).

19. F.D.M. Haldane, Phys. Rev. Lett. 67, 937 (1991).

20. Y. Wu, Phys. Rev. Lett. 73, 922 (1994).

21. A.M. Gavrilik and Y.A.Mishchenko, Ukr. J. Phys. 58, 1171 (2013).

22. A.M. Gavrilik and Y.A.Mishchenko, Nucl. Phys. B 891 , 466 (2015).

23. A. Rovenchak, Eur. Phys. J. B 87, 175 (2014).

24. A. Rovenchak, Phys. Rev. A 89, 052116 (2014).

25. P. F. Borges, H. Boschi-Filho, and C. Farina, Mod. Phys. Lett. A 14, 1217 (1999).

26. S. Mashkevich, J. Myrheim, and K. Olaussen, Phys. Lett. B 382, 124 (1996).

ISSN 2071-0194. Ukr. J. Phys. 2016. Vol. 61, No. 2 
27. A. Kristoffersen, S. Mashkevich, J. Myrheim, and K. Olaussen, Int. J. Mod. Phys. A 13, 3723 (1998).

28. A.P. Polychronakos, Phys. Lett. B 365, 202 (1996).

29. Q.A. Wang, Entropy 5, 220 (2003).

30. Y. Kaupp, S. Liraki, D. Tayurskii, A. Useinov, A. El Kaabouchi, L. Nivanen, B. Minisini, F. Tsobnang, A. Le Méhauté, and Q.A. Wang, J. Low Temp. Phys. 150, 660 (2008).

31. G. Kaniadakis, Physica A 296, 405 (2001).

32. G. Kaniadakis, Entropy 15, 3983 (2013).

33. R.T. Delves, G.S. Joyce, and I.J. Zucker, Proc. R. Soc. Lond. A 453, 1177 (1997).

34. A. Rovenchak, Fiz. Nizk. Temp. 39, 1141 (2013).

35. A.A. Rovenchak, Physics of Bose Systems (Ivan Franko National University of Lviv, Lviv, 2015) (in Ukrainian).

36. C. Tsallis, J. Stat. Phys. 52, 479 (1988).

37. V. Kac and P. Cheung, Quantum Calculus (Springer, New York, 2002)

38. Y. Yang, S. Xie, W. Feng, and X. Wu, Mod. Phys. Lett. A 13, 879 (1998).

39. A.M. Gavrilik and A.P. Rebesh, Mod. Phys. Lett. B 25 (2012).
40. Springer Handbook of Materials Measurement Methods, edited by H. Czichos, T. Saito, and L. Smith (Springer, Berlin, 2006).

Received 10.06.15.

Translated from Ukrainian by O.I. Voitenko

М.Я. Горнецъка, А.А. Ровенчак

\section{ДВОПАРАМЕТРИЧНІ МОДИФІКАЦІї}

СТАТИСТИК ЕНІОНІВ

$\mathrm{P}$ е $з$ ю м е

У роботі розглянуто двопараметричні моделі дробових статистик, які спрямовані на встановлення виразу для чисел заповнення вільних еніонів. Знайдено віріальні коефіцієнти для таких типів статистик: $\kappa$-деформовані статистики Поліхронакоса і Голдейна-Ву, модифіковані з qекспонентою статистики Поліхронакоса і Голдейна-Ву в бозонній границі, неповна та неадитивна статистика Джентіле для різних значень максимального заповнення рівня. Встановлено та проаналізовано зв'язок між різними типами дробових статистик та статистикою еніонів. 